\title{
Extraction of Genetic Networks (GN) Using Static Bayesian Belief Networks From Genome-Wide Temporal Microarray Data
} Judit Kumuthini*

\author{
Address: Cranfield University, Department of Analytical Science and Informatics (DASI) Cranfield University, Barton Rd, Silsoe, Bedfordshire, \\ MK45 4DT, UK. \\ Email: Judit Kumuthini* - S.j.kumuthini.s02@cranfield.ac.uk \\ * Corresponding author
}

from BioSysBio: Bioinformatics and Systems Biology Conference

Edinburgh, UK, I4-15 July 2005

Published: 2I September 2005

BMC Bioinformatics 2005, 6(Suppl 3):S8

The extraction of Gene Regulatory Network (GRN) for genome-wide microarray data is still at an early stage. In this project Bayesian belief networks (BBN), a graph based representation of joint probability distributions that capture the conditional dependencies between genes are applied to genome-wide E coli microarray data. The extracted GRN is found to be valid when compared with genome pathway databases such as KEGG and Ecocyc. In addition, a novel quantitative simulation technique was applied to the extracted BN to learn the marginal probabilities expression status of all the genes in the network.

We present GRNs, extracted using the Taboo and taboo order algorithms from temporal expression data for 4000 genes. Missing values were inferred using the EM algorithm. The extracted GRN shows strong similarity with the actual classification of the phases of yeast cell cycle. The architecture of individual pathway and inter pathway relation ship among these genes were largely preserved. Further, we also present the effect of different discritization class width of normalised Log2 expression ratios and the impact on the GRN architecture.

This GRN provides a richer model for analysing gene expression patterns, which captures the interaction among various genes in terms of probabilities and conditional dependencies. The results of this proof of concept study demonstrate that the BBN approach is well suited to modelling DNA microarray data, which is characterised by a high degree of measurement noise and variability. These results, and BBN's strengths in stochastic modelling and incorporation of prior knowledge, indicate potential for determining previously unknown or incomplete
GRNs. This work was supervised by Dr Conrad Bessant, Prof. Selly Saini. 